\title{
GAMA: The effect of environment on galaxy emission line properties
}

\author{
Oliver Steele ${ }^{1}$, Daniel Thomas ${ }^{1}$, Claudia Maraston ${ }^{1}$, \\ James Etherington ${ }^{1}$ and the GAMA collaboration \\ ${ }^{1}$ Institute of Cosmology and Gravitation, University of Portsmouth, Dennis Sciama Building, \\ Burnaby Road, Portsmouth, PO1 3FX, UK \\ email: oliver.steele@port.ac.uk
}

\begin{abstract}
We study the influence of environment on emission line properties using the Galaxy And Mass Assembly (GAMA) survey, taking care to disentangle the role of mass and environment. We look at the role of local density separating galaxies into classifications star forming, AGN, and SF/AGN composite using the BPT diagnostic diagram. We find that environment is generally less important as a driving factor than galaxy mass. The presence of emission lines, whether driven by star formation or central supermassive black hole activity mostly depends on galaxy mass consistently for all galaxy types.
\end{abstract}

Keywords. galaxies: active, galaxies: evolution, galaxies: general

\section{Introduction}

The impact of environment on galaxy evolution is a much debated and researched topic best visible through the well-known morphology-density relationship (e.g. Oemler 1974, Dressler 1980), through which there is a strong preference for comparable galaxy morphologies to appear in similar density environments. Studies into the importance of environment at a given morphology have proven indecisive, with some favouring environment as the major driving factor (e.g. Trager et al. 2000, Mendes de Oliveira et al. 2005) whilst others claim that mass is a more significant parameter (e.g. Thomas et al. 2010, Wake et al. 2005, Bundy et al. 2006).

Whilst some papers have investigated the impact of environment on star formation rates (SFR) (e.g. Peng et al. 2010) and generally found that it is negligible compared to that of mass, the impact on general emission line properties has not yet been studied in great detail.

In this paper we use the Galaxy And Mass Assembly (GAMA) survey to investigate the impact of environment on emission line properties.

\section{Data}

The GAMA survey is a multi-wavelength galaxy survey that upon completion will cover over 360 square degrees and consist of roughly 400,000 galaxies for $z \lesssim 0.4$ (Driver et al. 2011). It combines optical spectroscopy taken at the AAT with photometry taken at a variety of telescopes (CST, VISTA, Herschel, ASKAP and GALEX) ranging from radio to ultraviolet wavelengths. In Phase-I of GAMA, objects were spectroscopically observed up to an $r$-band magnitude limit of 19.4 for two of the GAMA fields, whilst the third field had a deeper 19.8 mag limit, all to a $98 \%$ completeness level. This depth makes it an excellent complement to SDSS as it probes down to lower stellar masses. A 
summary of the spectroscopic processing applied to the data can be found in Hopkins et al. (2013).

Our sample was created by combining $\sim 153,600$ objects spectroscopically observed by GAMA, with $\sim 15,000$ SDSS Data Release 7 objects that fall into the GAMA fields.

\section{Analysis Tools}

\subsection{Spectroscopic measurements}

We use the publicly available codes pPXF (Cappellari \& Emsellem 2004) and GANDALF (Sarzi et al. 2006) to calculate stellar kinematics and derive emission line properties. GANDALF fits stellar population templates and Gaussian emission line templates to the observed spectra simultaneously, allowing for the separation of stellar continuum and absorption from ionised gas emission, whilst pPXF extracts stellar kinematics from the data in pixel space. Dust reddening is accounted for by adopting a Calzetti (2001) obscuration curve and applying it to the data. Using this method we are able to extract stellar kinematics, gas kinematics, emission line fluxes and emission line equivalent widths (EWs) from the data.

We adopt the stellar population templates from Maraston \& Strömbäck (2011) based on the MILES stellar library (Sánchez Blázquez et al. 2006), extended in the blue for $\lambda<3500 \AA$ using theoretical spectra from the model of Maraston et al. (2009) based on the theoretical library UVBLUE (Rodríguez-Merino et al. 2005).

Details of this analysis tool and its calibration with SDSS data can be found in Thomas et al. (2013). A thorough presentation of the emission line measurements in GAMA is provided by Hopkins et al. (2013).

\subsection{Environment}

We calculated the Adaptive Gaussian Environment (AGE) Density of all of the galaxies in the sample with a redshift below 0.18 to define a pseudo-volume-limited population. See Schawinski et al. (2007a) and Thomas et al. (2010) for details on how this method was performed. We measured the environment for $\sim 37,000$ spectra.

\section{Emission line properties}

The equivalent widths and amplitude-over-noise $(\mathrm{AoN})$ values of the lines [OIII $] 5007$, $[\mathrm{NII}] 6583, \mathrm{H} \beta$ and $\mathrm{H} \alpha$ are measured for all objects with redshift below $\sim 0.45$, representing $96.8 \%$ of all objects. These lines allow for the usage of the well known Baldwin, Phillips and Terlevich (BPT) diagnostic diagram introduced in Baldwin, Phillips \& Terlevich (1981) and Veilleux \& Osterbrock (1987), and widely used in galaxy studies. This method compares the line ratios $[\mathrm{OIII}] / \mathrm{H} \beta$ and $[\mathrm{NII}] / \mathrm{H} \alpha$ to determine the ionisation source in galaxies.

We use the empirical separation between star forming galaxies and AGN from Kauffmann et al. (2003) and the theoretical extreme starburst line from Kewley et al. (2001) to identify pure star forming and pure AGN emission. We adopt the common practise of assuming the area between these lines is populated by galaxies with composite star forming and AGN spectra. We further use the dividing line defined by Schawinski et al. (2007b) to distinguish between LINER and Seyfert emission based on SDSS galaxy classifications obtained through the $[\mathrm{SII}] / \mathrm{H} \alpha$ ratio. We find that $18.4 \%$ of the total sample have all four BPT lines with AoN > 2, of which 54.2\% are classified as star forming, $28.7 \%$ as composite, $9.5 \%$ as Seyfert and $6.5 \%$ as LINER. 

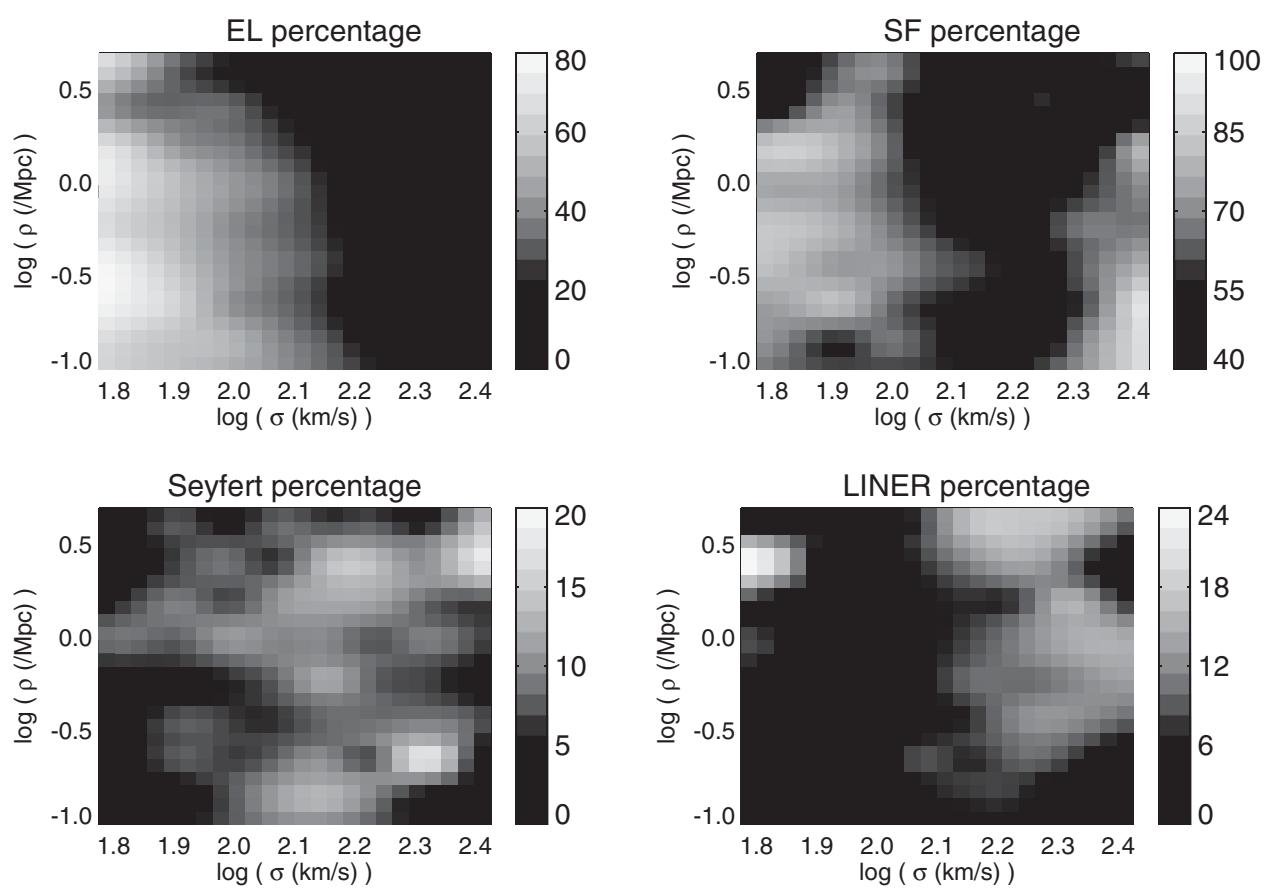

Figure 1. Percentage of emission line galaxies (top left panel) as a function of galaxy velocity dispersion and environmental density. The percent of star forming galaxies, Seyferts, and LINERs within the emission line population are shown by the top-left, bottom-left, and bottom-right panels, respectively. The high LINER fraction at very low sigma is an artefact caused by low number statistics, as is the high fraction of star forming galaxies at high sigma and low density.

For the purposes of this paper we consider galaxies with all four lines having a value of AoN > 2 as having emission lines, and all other galaxies as 'passive'.

\section{Results \& Discussion}

After applying cuts requiring that all objects investigated have $65 \mathrm{~km} \mathrm{~s}^{-1}<\sigma<$ $400 \mathrm{~km} \mathrm{~s}^{-1}, d \sigma / \sigma<0.3$ and $0 \mathrm{Mpc}^{-1}<\rho<50 \mathrm{Mpc}^{-1}$, we were left with 19,000 objects, of which $26 \%$ had emission lines.

Fig. 1 shows the percentage of emission line galaxies out of the whole sample, and star forming galaxies, composite galaxies, Seyferts and LINERs out of the emission line sample as functions of stellar velocity dispersion and local density. There are a few artefacts to note that were caused by low number statistics, specifically the high LINER percentage bubble at low sigma and the high star forming percentage at the high-sigma low-density corner.

We find that emission lines have a far stronger dependence on velocity dispersion (a proxy for galaxy mass) than environmental density. The top-left panel clearly shows that the emission line fraction decreases with increasing mass and only depends mildly on environment. There is a second order effect of slightly increasing emission line fraction with decreasing density at a given velocity dispersion.

Regarding specific BPT types, in general we find that none of the various emission line classes have a strong dependence on density, with differences being driven primarily by mass. Most of our sample consists of star forming galaxies at low velocity dispersion and across a wide range of densities. The fraction of star forming galaxies shows a similar 
pattern. The top-right panel shows that the fraction of star forming galaxies is highest at low galaxy masses and, again, only depends mildly on galaxy environment. The secondary fingerprint from the environment can be seen from a minimum of star forming galaxy fraction at high galaxy masses and environmental densities.

Finally, the bottom-right panel shows that the fraction of galaxies with LINER-like emission line properties again mostly depends on mass with the largest fraction of LINERs being found in massive galaxies, a trend largely independent of galaxy environment.

These results are in agreement with stellar population studies (e.g., Thomas et al. 2010), and show that galaxy mass is the primary driving factor of galaxy properties, with environmental density playing a secondary role.

These findings are inkeeping with current theory and observations, as late-type galaxies are more likely to have emission lines, are less massive and are found in less dense environments than their early-type counterparts, leading to a morphologically driven explanation for these results. A detailed analysis based on GAMA DR2 will be presented in a forthcoming paper (Steele et al., in preparation).

\section{Acknowledgments}

The Science, Technology and Facilities Council is acknowledged for their financial support. Numerical computations were done on the Sciama High Performance Computer cluster which is supported by the ICG, SEPNet and the University of Portsmouth.

\section{References}

Baldwin J. A., Phillips M. M., \& Terlevich R. 1981, PASP, 93, 5

Bundy K. et al. 2006, ApJ, 651, 120

Calzetti, D. 2001, PASP, 113, 1449

Cappellari M. \& Emsellem E. 2004, PASP, 116, 138

Dressler, A. 2000, ApJ, 236, 351-365

Driver S. P. et al. 2011, MNRAS, 413, 971

Hopkins A. M. et al. 2013, MNRAS, 430, 2047

Kauffman G., et al. 2003, MNRAS, 346, 1055

Kewley L. J., Dopita M. A., Sutherland R. S., Heisler C. A., \& Trevena J. 2001, ApJ, 556, 121

Maraston C., Nieves Colmenárez L., Bender R., \& Thomas D. 2009, A\&A, 493, 425

Maraston C. \& Strömbäck G. 2011, MNRAS, 418, 2785

Mendes de Oliviera C., Coelho P., González J. J., \& Barbuy B. 2005, AJ, 130, 55

Oemler, A., Jr. 1974, ApJ, 194, 1-20

Peng Y.-j. et al. 2010, ApJ, 721, 193

Rodríguez-Merino L. H., Chavez M., Bertone E., \& Buzzoni A. 2005, ApJ, 626, 411

Sánchez Blázquez P., Gorgas J., Cardiel N., \& González J. J. 2006, A\&\&A, 457, 809

Sarzi M. et al. 2006, MNRAS, 366, 1151

Schawinski K. et al. 2007, ApJS, 173, 512

Schawinski K., Thomas D., Sarzi M., Maraston C., Kaviraj S., Joo S.-J, Yi S. K., \& Silk J. 2007, MNRAS, 382, 1415

Thomas D., Maraston C., Schawinski K., Sarzi M., \& Silk J. 2010, MNRAS, 404, 1775

Thomas D., Steele O., \& Maraston C., et al. 2013, MNRAS, 431, 1383

Trager S. C., Faber S. M., Worthey G., \& González J. J. 2000, AJ, 120, 165

Veilleux S. \& Osterbrock D. E. 1987, ApJS, 63, 295

Wake D., Collins C. A., Nichol R. C., Jones L. R., \& Burke D. J. 2005, ApJ, 627, 186 